\title{
PULMONARY ALVEOLAR MICROLITHIASIS
}

\author{
BY \\ M. ABDEL-HAKIM, S. EL-MALLAH, M. HASHEM, AND - S. ABDEL-HALIM \\ From the Chest, Thoracic Surgery, and Pathology Departments, Cairo University, United Arab Republic
}

(RECEIVED FOR PUBLICATION APRIL 16, 1959)

Pulmonary alveolar microlithiasis is a rare disease of obscure nature characterized by intraalveolar calcific deposits that show radiologically as multiple small dots of calcification evenly scattered throughout the lungs. The striking feature about the disease is the diffuse and extensive radiological appearance in contrast to the minimal, or lacking, symptoms and signs early in the disease.

\section{INCIDENCE}

Eighteen cases have been reported in the literature, but the diagnosis was established during life in eight cases only. To our knowledge, the two cases we report are the nineteenth and twentieth.

Among these 18 cases it has been noticed that 11 were in men and seven in women. The youngest patient reported was 6 years old, but the average age was between 30 and 50 years.

Diagnosis was made at necropsy in 10 of the 18 cases, by pulmonary biopsy in four, and radiologically and clinically in four.

A familial incidence has been noticed in some instances. Mikhailov (1954) reported the disease in two brothers and in the son of one of them; the diagnosis was verified by lung biopsy in one of them, but the other two showed similar radiographic pictures. Our two cases are brothers aged 30 and 35 , and the diagnosis was confirmed during life by examining a biopsy obtained from the lung of one of them.

\section{Pathogenesis}

The condition was first described by Puhr in 1933, and as yet the aetiology is unknown. An abnormality of local calcium tissue metabolism in the lung has been postulated, but no disturbance in the systemic calcium metabolism has been demonstrated. It is supposed that the precipitation of calcium salts in the lung may be facilitated by the increase in $p \mathrm{H}$ of venous blood as a result of loss of carbonic acid.

The relationship of pulmonary alveolar microlithiasis to dust exposure deserves con- sideration, since six of the reported cases were exposed to mineral or fibre dust. Furthermore (Chinachoti and Tangchai, 1957), during a routine chest radiographic survey in Bangkok, Thailand, abnormal pulmonary shadows were seen in nine men who had been heavily addicted to the inhalation of snuff. These men were accustomed to insufflate powdered snuff into the nose at two- or three-hour intervals for 20 to 30 years. None had tuberculosis. One of them developed pulmonary symptoms and died; he was found to have pulmonary alveolar microlithiasis at necropsy. The snuff used in Thailand is a fine, dry, $\tan$ powder which is taken in small doses with a U-shaped metal tube. One end of the tube is inserted into the nostril and the other end into the mouth. A blast of air from the mouth disperses the powder into the nasal cavity, from where it is inspired suddenly. The snuff, on analysis, was found to be composed of $75 \%$ organic material, $15.4 \%$ silicates, and $1.6 \%$ silica. Its calcium content is $9.47 \%$. The low content of silica and the nature of pulmonary lesions excluded silicosis.

Further discussion of the aetiology of the disease led Kent, Gilbert, and Meyer (1955) to suggest that it is a "peculiar exudative response to a variety of insults which include pneumonia and rheumatic fever."

\section{Pathology}

The pleura is granular, blue black, and thickened or hard. Occasionally a small collection of blood-stained fluid is found in the pleural space. A grating sensation is felt on cutting through the lung. When a piece of lung is fixed in Zenker's solution and examined microscopically, concentrically laminated concretions are found in the alveoli affected by the disease. They stain deep blue by the haematoxylin-eosin-azure method. A foreign body giant cell response is a prominent feature. A moderate increase in interstitial fibrous tissue is noted, and the alveoli that contain concretions display fibrosis of 
their walls almost uniformly. A striking feature is the presence of numerous refractile colourless crystalline particles at the periphery of the concretions, between their lamellae, within the giant cells, or between the giant cells and calcific lamellae.

The alveolar septa are irregularly thickened and collagenous in patches and air spaces are obliterated. In some cases, mononuclear cells and dust cells were noted in some alveoli and interstitial tissue. Occasionally small patches of metaplastic osseous tissue replace a few alveoli and enclose several of the calcific bodies.

\section{Clinical Picture}

There are certain clinical and radiological features by which the disease can sometimes be diagnosed during life. The most outstanding observation is that the disease in its early stage manifests with few symptoms and even these may be absent altogether; this contrasts with the extensive radiological opacities seen in both lungs. In the later stages, progressive pulmonary insufficiency, cor pulmonale, and congestive heart failure appear.

The radiological picture, which was described in detail by Sosmàn, Dodd, Jones, and Pillmore (1957), is characterized by the diffuse fine pulmonary infiltrates that are less marked in the apices. The calcification is best seen under magnification of overexposed or bucky films. This picture has to be differentiated from that occasionally encountered in some cases of mitral stenosis, from pneumoconiosis, and from reticulosis. The history, clinical picture, other radiological findings, and E.C.G. will help to differentiate these conditions. A pulmonary biopsy settles the diagnosis.

\section{CASE REPORTS}

CASE 1.-A journalist, a man aged 30 , was not complaining of any symptoms, and it was during a routine examination that dense pulmonary infiltrates were discovered radiologically. He was admitted to hospital for investigation. His temperature was $37^{\circ}$ C. pulse regular at $90 /$ minute, respiration 32 /minute, and blood pressure $120 / 80 \mathrm{~mm}$. Hg. There was no clubbing of the fingers or enlargement of the superficial lymph nodes. Clinical examination did not reveal any abnormality of the heart or the abdomen, but over the chest an impaired note was elicited on percussing the left infrascapular area and a few fine crepitations were heard over both infrascapular zones.

The radiological picture of the chest (Fig. 1) showed bilateral dense infiltrates distributed throughout both lung fields, partially sparing the extreme apices and showing a homogeneous opacity involving the costo-

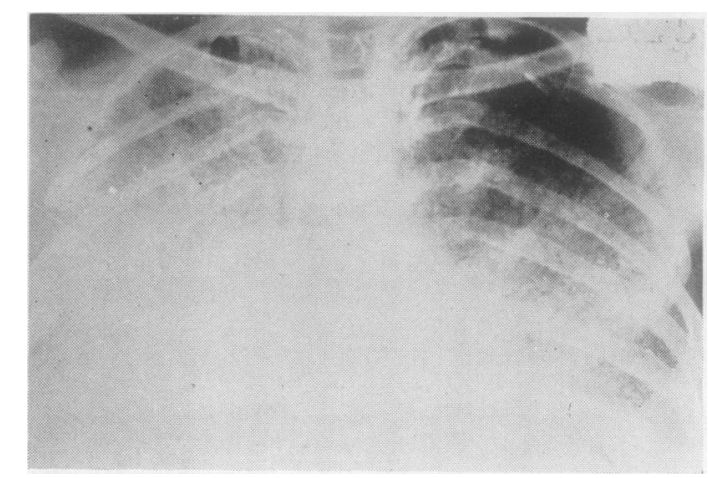

FIG. 1.-Radiograph of the chest of Case 1 showing the dense infiltrates occupying both lung fields, particularly the right lower zone.

phrenic angle, suggesting the possible presence of a small pleural effusion. Radiological examinations of the skull, long bones, and phalanges did not show any abnormality.

Sputum was repeatedly negative for tuberculosis. A tuberculin test was strongly positive. Blood analysis showed a normal picture except for a moderate eosinophilia $(12 \%)$. The blood sedimentation rate was 17 and $39 \mathrm{~mm}$. for the first and second hours using Westergren's method. Examinations of stools and urine were negative for any parasitic infestation. The Wassermann reaction was negative. Bone marrow examination by sternal puncture showed a total count of 148,000 with a normal differential picture.

Pulmonary function studies gave the following information:

Pulse $102 /$ minute, dropping to $88 /$ minute after lying down for 15 minutes; rate of breathing $32 /$ minute, dropping to $26 /$ minute after lying down for 15 minutes ; weight $61 \mathrm{~kg}$., height $173 \mathrm{~cm}$., and surface area $1.7 /$ square metre.

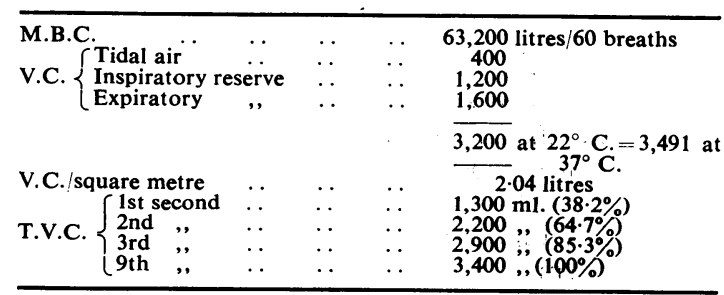

After an injection of $1 \mathrm{ml}$. adrenaline and $1 \mathrm{ml}$. ephedrine, the following results were obtained 20 minutes later:

\begin{tabular}{|c|c|c|}
\hline & $\begin{array}{c}\text { Total } \\
\text { Vital Capacity } \\
3,200 \mathrm{ml} .\end{array}$ & $\begin{array}{l}\text { Calculated } \\
\text { Vital Capacity } \\
\text { for } 3,400 \mathrm{ml} \text {. }\end{array}$ \\
\hline 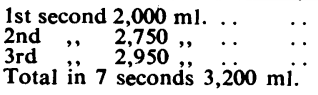 & $\begin{array}{l}62.5 \% \\
86 \% \\
92 \%\end{array}$ & $\begin{array}{l}60 \% \\
81 \% \\
89.7 \%\end{array}$ \\
\hline
\end{tabular}




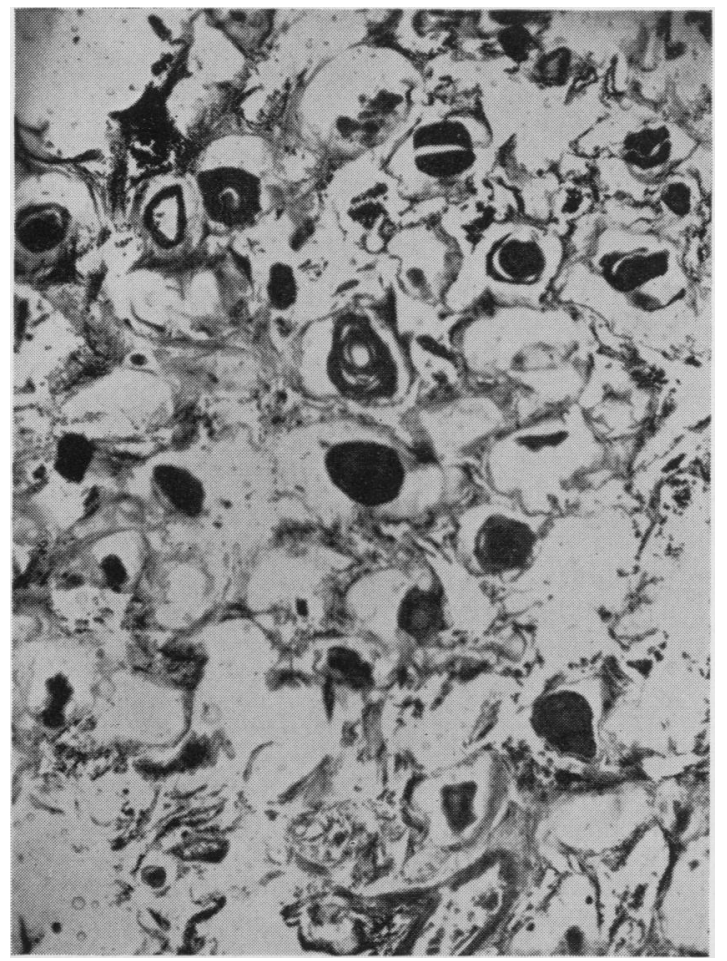

FIG. 2.-Section of lung biopsy of Case 1 showing the lung vesicles occupied by calcified concentrically lamellated bodies.

The patient could not give the total vital capacity as before the use of bronchodilators, probably because of fatigue.

Chemical studies showed that the serum calcium level was $8 \mathrm{mg} . / 100 \mathrm{ml}$, the serum phosphorus $8 \mathrm{mg} . / 100 \mathrm{ml}$, and the serum alkali phosphatase 8 units. The urine creatinine and creatine content levels in 24 hours were 1.178 and $0.305 \mathrm{~g}$.

There was no mitral stenosis and no history of exposure to dust inhalation or snuff. Pulmonary tuberculosis and Boeck's sarcoid were seriously considered. Hence, a combined treatment with streptomycin and isonicotinic hydrazide was tried for eight weeks without benefit. The result of treatment was assessed by comparison of radiographs, as the patient was not complaining of symptoms.

This treatment having failed, cortisone and vitamin $D_{2}$ in weekly doses of 600,000 units were tried. But the condition remained unchanged.

We believed then that lung biopsy was indicated and that it was the only means that would lead to the correct diagnosis ; but much argument was needed before we could persuade the patient, who had no symptoms, to undergo the operation.

The chest was opened through the fifth intercostal space by a lateral incision 5 in. long.

The pleural cavity was free of adhesions and there was no fluid. The oblique and transverse fissures were not adherent. The upper lobe was bluish and contained many soft cysts measuring $1-2 \mathrm{~cm}$. in diameter. It contained two hard nodules in its centre that were more or less smooth on the surface and each about $2 \mathrm{~cm}$. in diameter. The middle and lower lobes were pink, indurated, and felt gritty, with fine, small nodules like particles of sand that could be felt between the fingers. No glands were detectable in the hilum.

A small piece of lung tissue was removed from the lower edge of the upper lobe. No clamp could be applied on the lung owing to its induration. The knife was cutting through tough tissue as if passing through a very unripe pear. It was remarkable that no bleeding occurred from the cut edge and very little air leaked. The cut surface was sutured with several mattress sutures and the chest closed after inserting an underwater drain in the pleural cavity.

The post-operative period was stormy. The respiratory reserve of the patient was so much reduced that the temporary removal of the oxygen mask even after 48 hours led to cyanosis. However, after a further period and with breathing exercises he was weaned off the oxygen and his condition gradually returned to the pre-operative state.

On examining the piece of lung removed, the covering pleura was found thickened in patches by

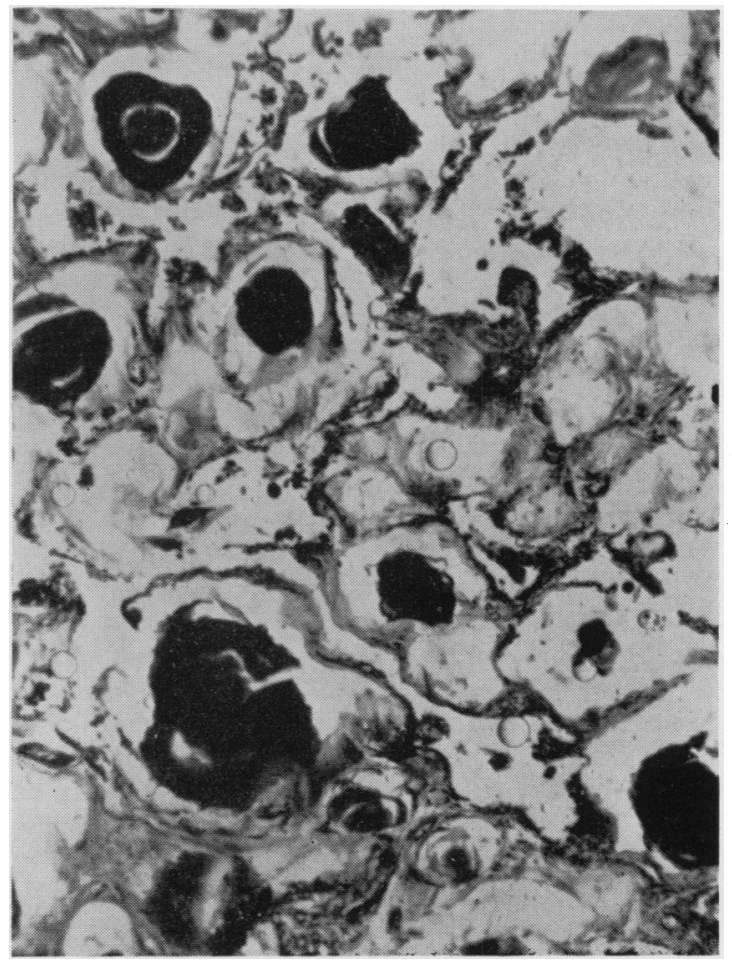

FIG. 3.-High-power view of the section seen in Fig. 2 showing the endarteritis obliterans of the interstitial vessels and histiocytes in some of the vesicles. 
hyaline fibrous tissue. The sections, examined histologically, showed that many of the lung vesicles were occupied by concentrically lamellated bodies resembling corpora amylacea, but they were calcified (Figs. 2 and 3). The calcified material took up the dark stain of haematoxylin-eosin and red stain by alizarin.

The vesicles were filled with homogeneous hyaline material, and in some of them in addition with a mononuclear inflammatory exudate around the periphery of the calcified bodies. Calcification appeared in some vesicles in the form of granules varying in size from 5 to $30 \mathrm{~m} \mu$. Histiocytes were seen occasionally in the vesicles, loaded with haemosiderin granules, the so-called heart failure cells.

Some of the alveolar ducts were lined by calcified material. Many of the interstitial vessels were markedly thickened by endarteritis obliterans.

The histological picture was thus consistent with pulmonary alveolar microlithiasis.

CASE 2.-A police officer, aged 35, the brother of the first patient, did not seek medical advice except when, on routine mass radiography of the chest, he was told about having "lung infiltrates."

He had no complaint and was enjoying good health; he was in the habit of playing games. The findings on clinical examination were similar to those of his brother. All investigations showed normal results except the chest radiographs, which revealed an exactly similar picture as his brother's.

The diagnosis was obscure until his brother came under our care and they agreed to a lung biopsy.

A radiograph of the chest was done for a third brother and did not show any abnormality.

\section{COMMENT}

In addition to the microliths seen in pulmonary alveolar microlithiasis intra-alveolar pulmonary concretions have been described in two forms:

Corpora amylacea in the lung were first described by Friedreich in 1856 (quoted by Finkbiner, Decker, and Cooper, 1957). They are rounded intra-alveolar bodies that may be laminated and may or may not stain as amyloid. They are not extensively calcified and are not visible radiologically. They are found in areas of emphysema, atelectasis, chronic pneumonitis, and infarction. They are not productive of pulmonary disease but are a manifestation of another disease. Cases of pulmonary alveolar microlithiasis differ from those in which numerous corpora amylacea occur, in that antecedent pulmonary disease is lacking in the former group and in the latter the intra-alveolar bodies are not visible radiologically as they are not extensively calcified.

Nodular pulmonary calcification and ossification occur in mitral stenosis. The distribution is similar to that in microlithiasis. The nodules in mitral stenosis tend to be larger and less numerous than in microlithiasis. Ossification is a prominent feature, whereas it is only occasionally encountered in microlithiasis.

Corpora amylacea may occur in the lung of a patient who has mitral stenosis; or a patient with morphologically acceptable microlithiasis may have mitral stenosis.

Our two patients are living in perfect health with no evidence of any insufficiency. There is no history of exposure to dust or inhalation of snuff. Their only habit is heavy smoking: more than 30 cigarettes daily for over 20 years, a practice which we believe has no relation to their disease.

The histopathological findings on examining the lung biopsy specimen may suggest that the calcified bodies have started in an inflammatory exudate. The presence of heart failure cells suggests that congestion has been present some time in the course of the disease. The thickened vessels of the stroma with endarteritis obliterans are a sign in favour of an old inflammatory condition, but the nature of an inflammation which could affect the lung in more than one member of a family, as was noticed in our cases and in some other instances, is interesting.

\section{SUMMARY}

Pulmonary alveolar microlithiasis is a rare disease characterized by diffuse pulmonary infiltrations seen on radiography in contrast to minimal symptoms and signs, particularly early in its course.

These infiltrations are caused by intra-alveolar calcified deposits.

Two case histories are reported. In the literature 18 cases have been reported, among which 10 have been proved histologically at necropsy and four by lung biopsy.

The two patients reported here are brothers and the diagnosis was confirmed by lung biopsy obtained from one of them.

Our thanks are due to Dr. A. Sami. Professor of Chest Diseases, Cairo University, for supplying the clinical data of Case 2 and the radiographs.

\section{REFERENCES}

Chinachoti, N., and Tangchai, P. (1957). Dis. Chest, 32, 687. Finkbiner, R. B., Decker, J. P., and Cooper, D. A. (1957). Amer. Rev. Tuberc., 75, 122

Friedreich, N. (1856). Virchows Arch. path. Anat., 9, 613.

Kent, G., Gilbert, E. S., and Meyer, H. H. (1955). A.M.A. Arch. Path., 60, 556.

Mikhailov, V.'(1954). Klin. med. (Mosk.), 32, No. 3, p. 31.

Puhr, L. (1933). Virchows Arch. path. Anat., 290, 156.

Sosmàn, M. C., Dodd, G. D., Jones, W. D., and Pillmore, G. U (1957). Amer. J. Roentgenol., T7, 947. 